\title{
Endoscopic third ventriculostomy in children with a fiber optic neuroendoscopy
}

\author{
Wenjun Shen ${ }^{1,2} \cdot$ Hasan R. Syed ${ }^{1} \cdot$ Gurpreet Gandhoke ${ }^{1} \cdot$ Roxanna Garcia $^{1} \cdot$ Tatiana Pundy $^{1} \cdot$ Tadanori Tomita $^{1}$ (D)
}

Received: 12 July 2017 / Accepted: 27 November 2017 / Published online: 16 December 2017

(C) The Author(s) 2017. This article is an open access publication

\begin{abstract}
Objective Endoscopic third ventriculostomy (ETV) provides a shunt-free treatment for obstructive hydrocephalus children. With rapidly evolving technology, the semi-rigid fiber optic neuroendoscopy shows a potential application in ETV by blunt fenestration. A retrospective analysis of our experience is reviewed.

Methods The authors review infants and children who underwent ETV using this technique from June 2004 to June 2016 with radiological and clinical follow-up done by a single surgeon. Patients who underwent ETV with channel scope were excluded. Demographic variables and operative reports were collected. Improvement of preoperative symptoms and avoidance of additional cerebrospinal fluid (CSF) diversion procedures were considered a success. The ETV success score (ETVSS) was used to correlate with clinical outcomes.

Results A total of 79 patients were included with a mean age of $8.3 \pm 5.5$ years, and $40.5 \%$ were female. The mean clinical and radiographic follow-up was $38.6 \pm 40.9$ months. The overall complication rate was $6.3 \%$, while $73.4 \%$ were considered successful. The ETV failure cases received conversion to ventriculoperitoneal shunt or redo of ETV with a median time of 2 months. The mean ETV success score was $74.3 \pm 11.8$ with positive correlation between success rate $(P<0.05)$. Kaplan-Meier failure-free survival rates of 30-day, 90-day, 6-month, 1-year, and 2-year were 89.9, 83.5, 78.5, 75.9, and 74.6\%. Eight patients required redo ETV, and five of these patients required eventual shunt placements. Approximately $61.9 \%$ of failure occurred within 3 months. Patients with post-intraventricular hemorrhage (IVH) /infection, and age younger than 12 months had the poorest outcome $(P<0.05)$.

Conclusions Blunt dissection of the third ventricle floor under endoscopic vision with the stylet tip of a fiber optic neuroendoscopy is safe and requires less equipment in the pediatric population. This technique is successful with an optimistic long-term outcome except for infants and the post-IVH and infectious subgroups.
\end{abstract}

Keywords Endoscopic third ventriculostomy $\cdot$ Hydrocephalus $\cdot$ Children $\cdot$ Surgical technique

\section{Introduction}

Endoscopic third ventriculostomy (ETV) is the most commonly performed endoscopic procedure in neurosurgery for obstructive hydrocephalus [1]. It provides a more physiological restoration of cerebrospinal fluid (CSF) between ventricular

Tadanori Tomita

ttomita@luriechildrens.org

1 Division of Pediatric Neurosurgery, Ann \& Robert H. Lurie Children's Hospital of Chicago, 225 E. Chicago Avenue, Chicago, IL 60611-2605, USA

2 Division of Pediatric Neurosurgery, Children's hospital of Fudan University, Shanghai, China cavities and the interpeduncular cistern. A prospective, multicenter study published in 2016 revealed the success rates of ETV of 6 months and 2 years are 66.7 and $57.8 \%$, respectively [2]. More and more centers around the world have used ETV to treat hydrocephalus. Kulkarni et al. first described the ETV success score (ETVSS) in 2009 [3], and this statistical model has shown validity and calibration in predicting ETV success within the first 6 months [2].

Although there is no consensus on the instrumentation and the technique to be used, the core is creating and widening the hole on the floor of the third ventricle. Rigid lens scopes with working channel provide the advantage of better visualization of anatomical structures, but are burdened by larger instrument size at the time of ventriculostomy. These rigid lens scopes with working channel are placed into the ventricle through a peel away catheter or trocar of $12.5 \mathrm{Fr}$. to $19 \mathrm{Fr}$. (4.1 to $6.0 \mathrm{~mm}$ ) 
in diameter. Several methods have been used to perform fenestration and its widening at the floor of the third ventricle, including mainly the alligator clamp, Fogarty balloon, laser, or water jet dissection $[4,5]$. In all of the abovementioned techniques, a working channel is required to allow for the use of the instrumentation. These techniques need larger burr hole for instrumentation, and leave larger needle tract. Although the use of instrumentation under endoscopic guidance claims to be safer because of the direct visualization of the instruments, vascular damage can nonetheless occur $[2,6]$.

In order to minimize the burr hole and needle tract, we have used regular sized ventricular catheter as a sheet for semi-rigid fiber optic neuroendoscopy for ETV. The semi-rigid fiber optic scope has $1.14 \mathrm{~mm}$ in diameter, and it is inserted into a ventriculostomy catheter of 2.3 to $2.8 \mathrm{~mm}$ in diameter. We present our results with the flexible neuroendoscope and blunt dissection technique for ETV in 85 patients.

\section{Methods}

\section{Patient selection and data source}

The authors conducted a retrospective analysis of infants and children who underwent ETV at Ann \& Robert H. Lurie Children's Hospital of Chicago from June 2004 to June 2016, which were performed with a uniform technique by a single surgeon (corresponding author). Hydrocephalus was untreated previously in all except for three patients with existing VP shunt. Among latter, ETV was performed at the VP shunt malfunction in two with a shunt removal and another without shunt revision. Demographic variables included gender, age at ETV, etiology of hydrocephalus, and prior shunt history. Operative reports were collected and the ETVSS were calculated. Patients were followed clinically and radiologically. All patients had one radiological study (CT or MR) in the early post-operative period and 1 month after the ETV. The subsequent follow-up was scheduled according to the clinical response and to the course of the etiology of the hydrocephalus. Success of ETV was determined on the basis of the last follow-up: the improvement of preoperative symptoms with or without reduction of ventricular size on radiological examination was considered a success, while the need for insertion of a VP shunt/redo of ETV or radiographic progression of hydrocephalus were considered a failure.

\section{Surgical technique}

A sterile $15-\mathrm{cm}$ semi-rigid $(1.14 \mathrm{~mm}$ in diameter) stylet neuroendoscope (Neuropen, Medtronic PS Medical, Goleta, CA, USA) is inserted into a ventricular slotted innervision catheter $(2.8 \mathrm{~mm}$ in outer diameter) with a distal slit tip (Innervision Ventricular Catheter, Medtronic PS Medical,
Goleta, CA, USA) (Fig. 1). All procedures were performed with the patient intubated, under general anesthesia, and in the supine position. A right frontal burr hole is placed at or just anterior to the coronal suture and $2-3 \mathrm{~cm}$ lateral to the sagittal midline usually on the right side.

After opening the dura, the neuroendoscope and ventricular catheter system are introduced into the frontal horn of the lateral ventricle using a free-hand technique or with the guidance of the neuro-navigation. Standard anatomical landmarks within the lateral ventricle are identified and the third ventricle is accessed through the ipsilateral foramen of Monro.

The floor of the third ventricle is inspected, the basilar artery may be identified by its transmitted pulsation, though sometimes it can be visualized through a thin floor. A stoma is placed in the center of the floor posteriorly to the vascular stain of the tuber cinereum and anteriorly to the mammillary bodies. The ependymal layer is first scored by the tip of the endoscope, and then identified the location by withdrawing the scope tip slightly. Once the scored site is satisfactory, the endoscope tip is advanced to perforate the pia. The careful advancement of the neuroendoscope allows us to visualize the progressive thinning of the floor and the eventual appearance of the pia. During the tip advancement, if reddish structures appear, further perforation is stopped, because it is likely heading to the basilar artery. During this time, the structures of the interpeduncular and prepontine cistern became visible through the transparent pia layer. Once the endoscope tip is in the cistern, the stoma is widened mechanically, by advancing the catheter over the endoscope further deeper into the prepontine space, and sliding the catheter sideways over the clivus. In the subarachnoid space, the Liliequist's membrane is fenestrated in a similar fashion. The prepontine space is carefully inspected in order to break any arachnoidal adhesions mechanically. After withdrawing the scope and the catheter into the third ventricle, the stoma is easily visualized (Fig. 2). Intraoperatively, the third ventriculostomy is judged successful if the edges of the stoma flap freely with the passage of CSF, and the basilar artery, its branches, and the belly of the

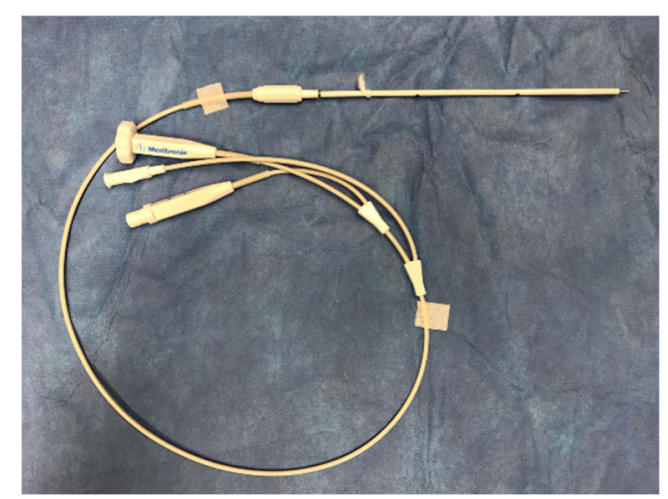

Fig. 1 NeuroPen fiber optic neuroendoscopy inserted into a ventricular slotted innervision catheter 


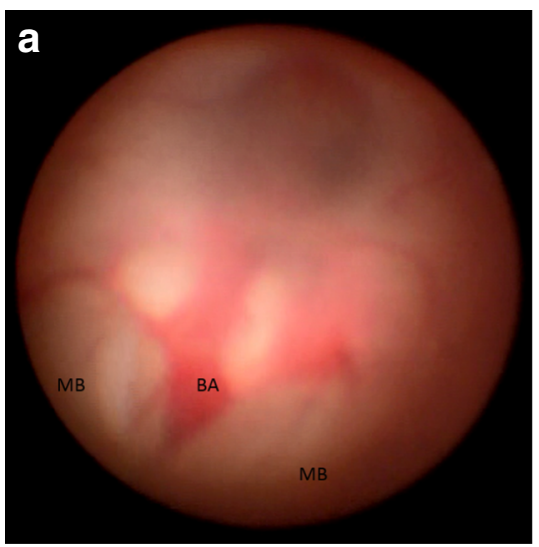

Fig. 2 a, b Surgical views of the floor of the third ventricle through a NeuroPen endoscope in a 4-year-old girl with diffuse intrinsic pontine glioma and obstructive hydrocephalus, before (a) and after (b) endoscopic third ventriculostomy (ETV). The basilar artery (BA) and

pons are visualized without any overlying membrane. Though there is no validated way to measure the size of the stoma, a good intraoperative reference is the diameter of the basilar artery or half a distance between the tuber cinereum and the anterior mammillary line, which are considered to be sufficient size of the stoma.

The ventricular catheter is routinely left in the lateral ventricle and connected to the Ommaya reservoir in the subgaleal space postoperatively.

\section{Statistical analysis}

Continuous variables were expressed as the mean \pm standard errors of the mean (SEM) and categorical variables are shown as raw values with percentages. Failure-free survival and curves were estimated using the Kaplan-Meier method and compared with the log-rank test (Mantel-Cox). $P$ values < 0.05 were considered statistically significant. All analyses were performed using the SPSS Version 19.0 software.

\section{Results}

A total of 85 ETV cases' meet inclusion criteria but 6 patients were lost to follow-up due to inadequate postoperative information. The characteristics of the $79 \mathrm{ETV}$ follow-up cases are listed in Table 1. The mean age is $8.3 \pm 5.5$ years old ( 2 month to 20 years old), and the male-to-female ratio was 1.5 . The predominant etiology for hydrocephalus was tumor (47 cases, $59.5 \%$ ) (Table 2), followed by aqueductal stenosis (10 cases, $12.9 \%$ ), unbiopsied tectal lesion ( 9 cases, $11.4 \%$ ), postintraventricular hemorrhage (IVH) (3 cases, 3.8\%), myelomeningocele ( 2 cases, $2.5 \%$ ), post-infection (1 case, $1.3 \%$ ), and other causes in the remaining 7 cases $(8.9 \%)$.

Eight patients required redo ETV, three redos in 1 child, and a single redo in 7 children, thus accounting for a total of

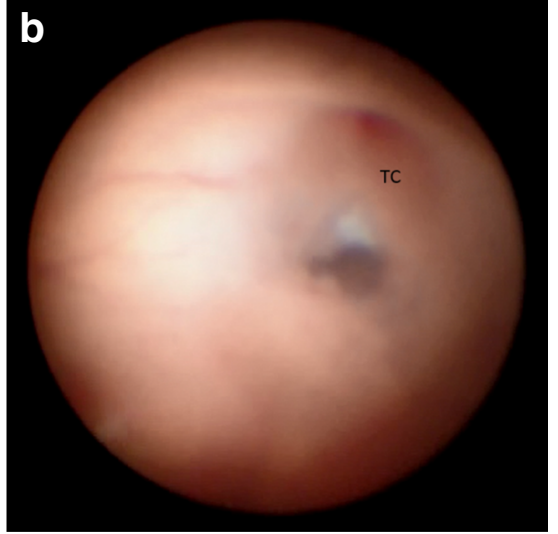

hypertrophic belly of the pons are visible between the mammillary bodies (MB) through semitransparent floor (a). A stoma after ETV is shown behind the vascular stain of the tuber cinereum (TC) (b). Note a rapid thickening of the floor of the third ventricle after ETV

95 procedures among 85 patients. The overall complication rate was $6.3 \%$ ( 6 out of 95 procedures): 1 intraoperative venous hemorrhages required a temporary external ventricular drainage (EVD), 2 intraoperative minor hemorrhages without EVD, and 3 complicated by postoperative staph ventriculitis.

Table 1 Summary of characteristics of patients

\begin{tabular}{ll}
\hline Variable & No. of cases $(\%)$ \\
\hline Previous shunt & $3(3.8 \%)$ \\
Age at ETV & \\
$<1$ month & $0(0.0 \%)$ \\
1 to $<6$ months & $4(5.1 \%)$ \\
6 to $<12$ months & $7(8.9 \%)$ \\
1 to $<10$ years & $33(41.8 \%)$ \\
$\geq 10$ years & $35(44.3 \%)$ \\
Etiology & \\
Tumor & $47(59.5 \%)$ \\
Aqueductal stenosis & $10(12.7 \%)$ \\
Tectal lesion & $9(11.4 \%)$ \\
Post-IVH & $3(3.8 \%)$ \\
Myelomeningocele & $2(2.5 \%)$ \\
Post-infection & $1(1.3 \%)$ \\
Other & $7(8.9 \%)$ \\
ETVSS & \\
10 & $0(0.0 \%)$ \\
20 & $1(1.3 \%)$ \\
30 & $0(0.0 \%)$ \\
40 & $1(1.3 \%)$ \\
50 & $2(2.5 \%)$ \\
60 & $6(7.6 \%)$ \\
70 & $29(36.7 \%)$ \\
80 & $28(35.4 \%)$ \\
90 & $12(15.2 \%)$ \\
\hline & \\
0 &
\end{tabular}


Table 2 Tumor etiology of hydrocephalus

\begin{tabular}{lll}
\hline Anatomy region & Etiology & $N=47$ \\
\hline Pineal region & & $N=8$ \\
& Pineoblastoma & 4 \\
& Germinoma & 2 \\
& AT/RT & 1 \\
& Teratoma & 1 \\
Cerebellar + 4th ventricle + CPA & & $N=16$ \\
& Medulloblastoma & 8 \\
& Ependymoma & 4 \\
& LGG & 2 \\
& Diffuse hystiocytosis & 1 \\
& Lymphoma & 1 \\
Mid brain + brainstem + thalamus & & $N=22$ \\
& LGG & 10 \\
& HGG & 5 \\
& DIPG & 4 \\
& NF & 2 \\
& PNET & 1 \\
& & $N=1$ \\
& LGG & 1 \\
& &
\end{tabular}

$A T / R T$ atypical teratoid/rhabdoid tumor, $C P A$ cerebellopontine angle, $L G G$ low-grade giloma, $H G G$ high-grade giloma, $D I P G$ diffuse intrinsic pontine glioma, $N F$ neurofibroma, $P N E T$ primitive neuroectodermal tumors

Five out of six patients with complications required the placement of a VP shunt eventually.

The mean follow-up time is $38.6 \pm 40.9$ months (range $1-$ 149 months), with approximately $73.4 \%$ technique success. Twenty-one cases (26.6\%) had ETV failure and were converted to VP shunt or had ETV redo with a median time of 2 months (range 1-48 months). The causes of failure were explored when redo or VP shunt with an endoscopy in 19 patients (summarized in Table 3). Most of the failure cases (13 cases, $61.9 \%$ ) occurred in the early post-operative period within 3 months. One patient had acute neurological deterioration with bradycardia 19 months after the ETV, which we considered late ETV failure. The mean age among ETV

Table 3 ETV failure reasons

\begin{tabular}{ll}
\hline Reasons & $N$ \\
\hline Stoma closure & 7 \\
Communicating hydrocephalus & 4 \\
Lilliquist membrane & 2 \\
Tumor progression & 2 \\
Subdural CSF collection & 2 \\
Infection & 1 \\
Ventricle abnormality & 1 \\
N/A & 2 \\
\hline
\end{tabular}

failure cases was significantly younger than shunt-free cases $(5.5 \pm 5.3$ vs $9.4 \pm 5.2, P<0.01)$. Patients with ETV due to post-IVH or infectious hydrocephalus had poorer outcome, only $25 \%$ success rate, and children younger than 12 months only have a $27.3 \%$ technique success rate (Table 4 ). Only one of three patients with existing shunt failed after ETV. The success rate for tumor, tectal lesion, and aqueductal stenosis is $80.4,77.8$, and $70.0 \%$, respectively (Table 4). Follow up MR show various degree of reduction in the ventricle size, but none showed slit-like ventricle (Fig. 3).

The mean ETVSS in all patients was $74.3 \pm 11.8$ (Table 5). There was a correlation between high ETVSS and technique success $(P<0.05)$. The area under receiver operator characteristic curve for ETVSS as an independent predictor of successful outcome was 0.72 (95\% CI $0.57-0.87$, sensitivity $98.3 \%$, specificity $17.7 \%$ ). Kaplan-Meier failure-free survival rates of 30-day, 90-day, 6-month, 1-year, and 2-year were 89.9, 83.5, $78.5,75.9$, and $74.6 \%$, respectively. Figure 4 shows the Kaplan-Meier survival curve for time to ETV failure.

\section{Discussion}

To access the ventricular system endoscopically, normal brain parenchyma needs to be traversed. Minimizing this collateral damage is the main theme behind using this minimally invasive technique. Although the rigid lens scopes provide highquality images and angled-variety, the advances in the field of fiber optics have led to significant improvements in image resolution. They can be inserted into the lumen of ventricular catheter without trocar or peelaway catheter which needs a larger burr hole and needle tract. The use of ventricular catheter as a sheet for ventricoscope is particularly useful when the ventricles are small or at the time of shunt revision. The endoscopic procedure is easily performed by a single surgeon without using any endoscope holder or requiring to tackle various other simultaneous instruments. Being semi-rigid, the fiber scope gives the surgeon the opportunity to bend in small angles and adjust to respective target trajectory with precision under the tip of the scope if necessary.

Moreover, when a patent stoma is achieved and widened by means of perforating instruments like forceps, the tip of the

Table 4 Incidence of success/failure according to etiology and age

\begin{tabular}{lllll}
\hline Diagnosis & All patients & Success & Failure & $p$ \\
\hline Tumor & 56 & $45(80.4 \%)$ & $11(19.6 \%)$ & 0.029 \\
Tectal lesion & 9 & $7(77.8 \%)$ & $2(22.2 \%)$ & 0.753 \\
Aqueductal stenosis & 10 & $7(70.0 \%)$ & $3(30.0 \%)$ & 0.793 \\
Post-IVH/infection & 4 & $1(25.0 \%)$ & $3(75.0 \%)$ & 0.024 \\
Infant (<12 months) & 11 & $3(27.3 \%)$ & $8(72.7 \%)$ & $<0.001$ \\
\hline
\end{tabular}


Fig. 3 An 8-year-old female presented with headaches and papilledema. Preoperative T2weighted MR (a) shows a tectal plate lesion and obstructive hydrocephalus. T2-weighted MR (b), 7 years after ETV, shows a reduction of the ventricles with flow void at the floor of the third ventricle

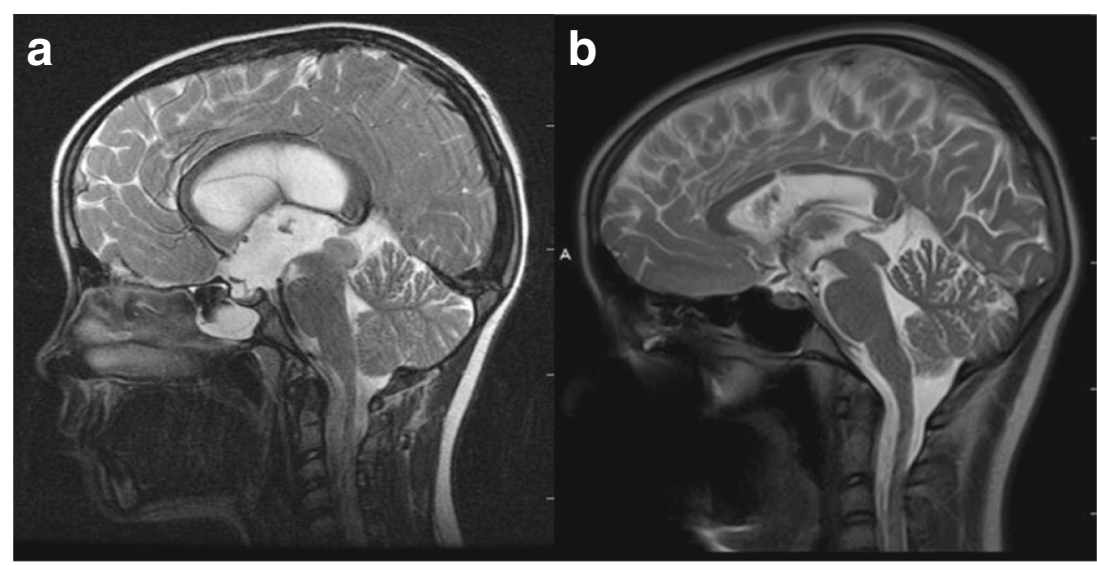

Fogarty catheter, or electrocautery, a working channel is essential. Injury to perforating vessels has been reported in such ways [6]. At the time of using the fiber neuroendoscope during the fenestration of the floor, one should be familiar with the anatomy of the floor of the third ventricle and avoid any reddish structure during the advancement of the tip of the endoscope during the perforation of the floor. The safest way is initially scoring the ependymal layer of the floor of the third ventricle by the tip of the endoscope to identify the location and direction of intended ETV.

The diameter of the scope has been linked to post-operative subdural collections in young children [7], and is also crucial to reduce the risk of damage to neural structures within the ventricles, such as the fornix, particularly in the presence of distorted anatomy like a scarred foramen of Monro [8] encountered in cases of children with history of prior shunt. It has been described that ETV has a 5 19\% complication rate containing venous hemorrhage, basilar artery perforation, subarachnoid or intraventricular hemorrhage, thalamic or hypothalamic contusion, fornix or midbrain injury, memory deficit, diabetes insipidus, weight gain, precocious puberty, bradycardia, hemiparesis, 3rd nerve palsy, meningitis, ventriculitis, superficial infection, CSF leak, subdural CSF collection, altered consciousness, and herniation syndrome [9-11]. We have a $6.3 \%$ complication rate without any injury to the endocrinological, neural, or vascular structures. Limiting and reducing complications are essential, as the incidence of failure of ETV is related to early complications as noted in our previous experience [12]. The present analysis validates this assumption,

Table 5 Relationship between ETVSS and failure-free survival

\begin{tabular}{lcc}
\hline ETVSS & $N$ & Failure-free survival (\%) \\
\hline$\leq 40$ & $2(2.6 \%)$ & 0 \\
$50-70$ & $37(46.8 \%)$ & 70.3 \\
$\geq 80$ & $40(50.6 \%)$ & 80.0 \\
\hline
\end{tabular}

because all procedures complicated by intraoperative hemorrhage or early post-operative infection ultimately lead to the placement of a VP shunt $(P<0.05)$. Our cohort has a similar mean ETVSS when compared to multicenter data [2] (74.3 vs 74.8) with no bias in the candidates selection. The comparison of 2-year failure-free survival rates between our series and multicenter data, 74.6 vs $57.8 \%$ respectively $(P<0.05)$, indicates certain advantages with fiber optic neuroendoscopy blunt dissection.

Although there is no way to measure the size of the stoma intraoperatively, the size of stoma is expected to correlate with the success of ETV: Kombogiorgas and Sgouros measured the relative size of stoma as the percentage of stoma diameter to the distance percentage of stoma between posterior clinoid and basilar artery. They reported that stoma size may correlate with success without statistical significance [13]. We achieve an improved stoma size by advancing the catheter over the endoscope and then sliding neuroendoscope sideways between the basilar artery and the clivus. Thick ventricular floor

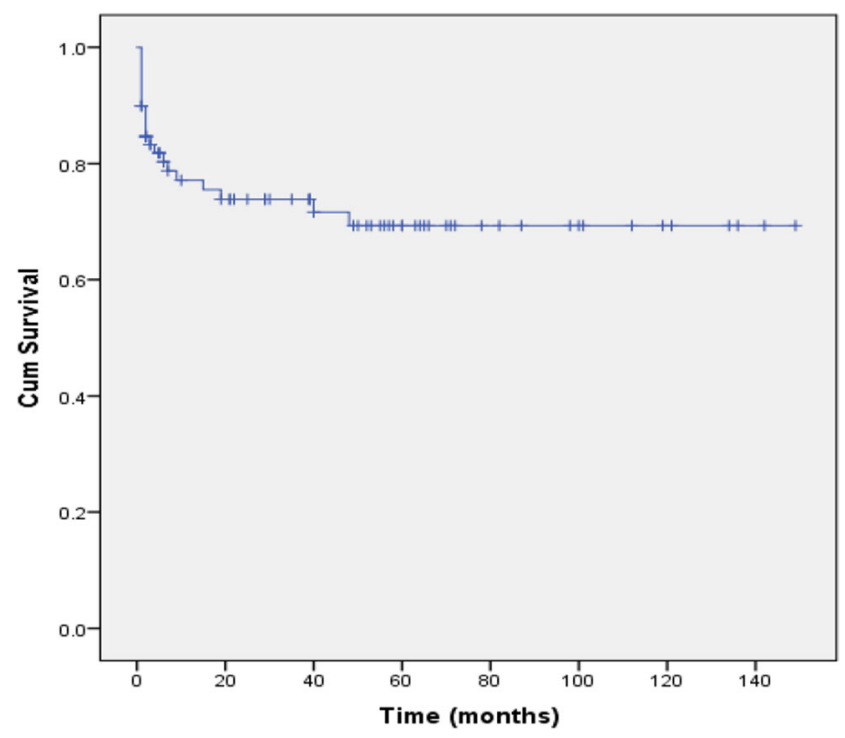

Fig. 4 ETV failure-free survival curve 
or arachnoid adhesion were considered one of the negative intraoperative findings for poor ETV outcome [14]. This technique allows to easy conversion to shunt in the event that intraoperative findings do not favor an ETV. We excluded four such intention failure cases from this study who had placement of VP shunt after aborting ETV. These patients represent $4.5 \%$ out of 89 ETV intentions. Promising data has been shown by the use of the FIESTA sequence of the MR in the preoperative assessment of the subarachnoid space, which will be useful in selecting favorable patients for ETV [15]. There is little information in the literature about ETV redo; while one paper from Germany in adult reported an $87.5 \%$ redo success rate [16], we have a $37.5 \%$ redo successful rate from our cohort of eight patients.

The success rate of this minimally invasive procedure was $73.4 \%$ in our series and is comparable to the other studies [2, 17]. Several factors have been shown to affect the outcome of ETV, in particular the age of patient at the time of operation, the cause of hydrocephalus, and prior shunting, which are factors that are used to calculate the ETVSS [2,3]. Our previous paper on ETV has confirmed relative lower success rate in younger children [18]. We found that IVH and infection were related to a negative outcome statistically, which is similar with the literature [2]. Radiological and intraoperative factors predictive of failure have also been reported [14, 19-21]. The pulsation of the floor is considered critical because it provides an indirect measure of the subarachnoid space, which indicates brisk CSF flow from the ventricle to the prepontine space [13]. Visualization of a naked basilar artery is a positive predictor of ETV success [2]. We were able to clearly identify the basilar artery in every case, only two failure cases had remaining membrane confirmed at the redo ETV (Table 3). The present experience shows that triventricular hydrocephalus secondary to tumor obstruction has more benefit from ETV (Table 4). The aqueductal stenosis cases had a 70.0\% success rate, while it is comparable to above $60 \%$ reported in the literatures $[22,23]$. Interestingly, in neoplastic cases, the success rate increased to $80.4 \%(P<0.05)$. Although 15 tumor progression cases had shorter follow-up time (14.2 \pm 16.8 months), patients with neoplastic cases as a whole had similar follow-up time compared to other etiology. After excluding this bias, our result confirms that obstructive hydrocephalus secondary to tumors either in pineal or posterior fossa regions is a good indication for ETV, as confirmed with the other reports [24-26]. On the contrary, if the tumor occupied the floor of the third ventricle or the prepontine cistern [27], we found a $33.3 \%$ (one out of three) success rate in this subgroup. While confounded by a small sample size, we believe pathology in this region is a contraindication to ETV.

The majority of ETV failure occurred in early postoperative period $[2,28] ; 13$ out of $21(61.9 \%)$ within the first 3 months and 17 out of $21(81 \%)$ within the first 6 months in our series. The cause of late rapid deterioration of ETV has not been fully studied, and it usually happened 2.5 years on average after initial ETV [29]. A population-based analysis showed that a second round of failure occurred around 3 years after the initial ETV procedure in children between 1 and 10 years of age with tumor or aqueductal stenosis [30]. We had one late rapid ETV failure at 19th month, but a second failure peak could not be found in our cohort. A total of 11 cases died from tumor progression in the follow-up period (mean follow-up time 14.2 \pm 16.8 months), which is statistically shorter than the other subgroups $(P<0.05)$. The poor survival prognosis in this subgroup may deprive the chance to reflect the second failure round.

In this series, nearly every patient had an Ommaya reservoir connecting to ventricular catheter following ETV, which allows us to gain access to the intracranial CSF, to monitor ICP and to drain the CSF if necessary. Moreover, potentially catastrophic acute hydrocephalus after ventriculostomy closure can be managed with a ventricular access device in place $[29,31,32]$. Radiological evidence of patent stoma could be achieved by injection contrast through the reservoir. The most failure reason is stoma closure due to the scaring, counting $33.3 \%$ ( 7 out of 21 ) of all the failure cases (Table 3 ), which is similar to a $42.8 \%$ rate reported in the literature [16]. The other failure reasons were combined communicating hydrocephalus, Liliequist membrane, tumor progression, subdural CSF collection, infection, and ventricle abnormality (Table 3).

The size of the stoma created at the floor of the third ventricle may affect the ETV survival. The larger the soma, the lower the failure rate would be [2]. The normal aqueduct of Sylvius is quite narrow. The ranges of luminal area of cerebral aqueduct in children was reported from $0.15 \mathrm{~mm}^{2}$ with a mean size of $0.5 \mathrm{~mm}^{2}$, while its length ranges from $12.8 \mathrm{~mm}$ at birth to $18 \mathrm{~mm}$ after 8 years old [33]. We have used a slotted innervision catheter rather than regular catheter as it provided a larger diameter [12]. Slotted innervision catheter with an outer diameter of $2.8 \mathrm{~mm}$ produces a surface area of a hole of $6.15 \mathrm{~mm}^{2}$ by a single puncture, whereas regular innervision catheter was $2.5 \mathrm{~mm}$ and $4.9 \mathrm{~mm}^{2}$, respectively. So our technique has provided adequate patency mechanically, much larger than the physiological size of the aqueduct. However, the difference between the normal cerebral aqueduct and the ETV stoma is that the former is lined by normal ependymal and the later has glial tissue and leptomeninges in the surrounding tissue. Therefore, over time the ETV can be closed due to the development of meningocerebral cicatrix, while the intact ependymal layer does not adhere under normal condition, which attributes to our one-third redo cases.

Studies have revealed that the ETV success rate is highly dependent on age $[2,3,18]$, we also found a statistic younger age in all the failure cases. The recent prospective comparison from the International Infant Hydrocephalus Study showed that infants $(<24$ months old) with aqueductal stenosis would benefit more likely from a shunt than ETV [34]; the actual 
success rates for ETV versus shunt at 3, 6, and 12 months were as follows: 68 vs. $95 \%, 66$ vs. $88 \%$, and 66 vs. $83 \%$. The infants in our cohort had a $27.3 \%$ chance of ETV success, while the risk of failure increased for ages younger than 6 months with a $25 \%$ chance, both consistent with the other reports $[34,35]$. As the initial outcome of treating young infant with ETV/choroid plexus coagulation (CPC) is promising [36], some recommend ETV/CPC as the first line of treatment for infants' hydrocephalus [37].

\section{Conclusions}

Blunt dissection of the third ventricle floor under endoscopic vision with the stylet tip of a fiber optic neuroendoscopy to perform ETV is safe, cost-effective, and requires less equipment in experienced hands. Inability of execution of ETV was $4.5 \%$ among attempted. This technique is successful with an optimistic long-term outcome but may be challenging in certain populations, including infants and the post-IVH and infectious subgroups.

Acknowledgements Wenjun Shen, M.D. was a visiting scholar from, and supported by Children's Hospital of Fudan University, Shanghai, China.

\section{Compliance with ethical standards}

\section{Conflict of interest None.}

Open Access This article is distributed under the terms of the Creative Commons Attribution 4.0 International License (http:// creativecommons.org/licenses/by/4.0/), which permits unrestricted use, distribution, and reproduction in any medium, provided you give appropriate credit to the original author(s) and the source, provide a link to the Creative Commons license, and indicate if changes were made.

\section{References}

1. Schroeder HW, Oertel J, Gaab MR (2008) Endoscopic treatment of cerebrospinal fluid pathway obstructions. Neurosurgery 62(Suppl 3):1084-1092

2. Kulkarni AV, Riva-Cambrin J, Holubkov R, Browd SR, Cochrane DD, Drake JM, Limbrick DD, Rozzelle CJ, Simon TD, Tamber MS, Wellons JC III, Whitehead WE, Kestle JRW, for the Hydrocephalus Clinical Research Network (2016) Endoscopic third ventriculostomy in children: prospective, multicenter results from the Hydrocephalus Clinical Rsearch Network. J Neurosurg Pediatr 18(4):423-429. https://doi.org/10.3171/2016.4.PEDS163

3. Kulkami AV, Riva-Cambrin J, Browd SR (2011) Use of the ETV success score to explain the variation in reported endoscopic third ventriculostomy success rates among published case series of childhood hydrocephalus. J Neurosurg Pediatr 7(2):143-146

4. Devaux BC, Joly LM, Page P, Nataf F, Turak B, Beuvon F, Trystram D, Roux FX (2004) Laser-assisted endoscopic third ventriculostomy for obstructive hydrocephalus: technique and results in a series of 40 consecutive cases. Lasers Surg Med 34(5):368-378. https://doi.org/10.1002/lsm.20045

5. Yadav YR, Shenov R, Mukerji G, Parihar V (2010) Water jet dissection technique for endoscopic third ventriculostomy minimises the risk bleeding and neurological complications in obstructive hydrocephalus with a thick and opaque third ventricle floor. Minim Invasive Neurosurg 53(4):155-158. https://doi. org/10.1055/s-0030-1263107

6. Schroeder HW, Gaab MR (1999) Intracranial endoscopy. Neurosurg Focus 6(4):e1

7. Wiewrodt D, Schumacher R, Wagner W (2008) Hygromas after endoscopic third ventriculostomy in the first year of life: incidence, management and outcome in a series of 34 patients. Childs Nerv Syst 24(1):57-63. https://doi.org/10.1007/s00381-007-0407-5

8. Benabarre A, Ibáñez J, Boget T, Obiols J, Martínez-Aran A, Vieta E (2001) Neuropsychological and psychiatric complications in endoscopic third ventriculostomy: a clinical case report. J Neurol Neurosurg Psychiatry 71(2):268-271. https://doi.org/10.1136/ jnnp.71.2.268

9. Schroeder HW, Niendorf WR, Gaab MR (2002) Complications of endoscopic third ventriculostomy. J Neurosurg 96(6):1032-1040. https://doi.org/10.3171/jns.2002.96.6.1032

10. Hellwig D, Grotenhuis JA, Tirakotai W, Riegel T, Schulte DM, Bauer BL, Bertalanffy H (2005) Endoscopic third ventriculostomy for obstructive hydrocephalus. Neurosurg Rev 28(1):1-34; discussion 35-38. https://doi.org/10.1007/s10143-004-0365-2

11. Bouras T, Sgouros S (2011) Complications of endoscopic third ventriculostomy. J Neurosurg Pediatr 7(6):643-649. https://oi. org/10.3171/2011.4.PEDS10503

12. Navarro R, Gil-Parra R, Reitman AJ, Olavarria G, Grant JA, Tomita $\mathrm{T}$ (2006) Endoscopic third ventriculostomy in children: early and late complications and their avoidance. Childs Nerv Syst 22(5): 506-513. https://doi.org/10.1007/s00381-005-0031-1

13. Kombogiorgas D, Sgouros S (2006) Assessment of the influence of operative factors in the success of endoscopic third ventriculostomy in children. Childs Nerv Syst 22(10):1256-1262. https://doi.org/10. 1007/s00381-006-0072-0

14. Greenfield JP, Hoffman C, Kuo E, Christos PJ, Souweidane MM (2008) Intraoperative assessment of endoscopic third ventriculostomy success. J Neurosurg Pediatr 2(5):298-303. https://doi.org/10.3171/PED.2008.2.11.298

15. Warf BC, Campbell JW, Riddle E (2011) Initial experience with combined endoscopic third ventriculostomy and choroid plexus cauterization for post-hemorrhagic hydrocephalus of prematurity: the importance of prepontine cistern status and the predictive value of FIESTA MRI imaging. Childs Nerv Syst 27(7):1063-1071. https://doi.org/10.1007/s00381-011-1475-0

16. Hellwig D, Giordano M, Kappus C. Redo third ventriculostomy (2013) World Neurosurg 79(2 Suppl):S22.e13-20. doi: https://doi. org/10.1016/j.wneu.2012.02.006

17. Di Rocco C, Cinalli G, Massimi L, Spennato P, Cianciulli E, Tamburrini G (2006) Endoscopic third ventriculostomy in the treatment of hydrocephalus in pediatric patients. Adv Tech Stand Neurosurg 31:119-219. https://doi.org/10.1007/3-211-32234-5_4

18. Ogiwara H, Dipatri AJ Jr, Alden TD, Bowman RM, Tomita T (2010) Endoscopic third ventriculostomy for obstructive hydrocephalus in children younger than 6 months of age. Childs Nerv Syst 26(3):343-347. https://doi.org/10.1007/s00381-0091019-z

19. Dlouhy BJ, Capuano AW, Madhavan K, Torner JC, Greenlee JD (2012) Preoperative third ventricular bowing as a predictor of endoscopic third ventriculostomy success. J Neurosurg Pediatr 9(2): 182-190. https://doi.org/10.3171/2011.11.PEDS11495

20. Greenfield JP, Hoffman C, Kuo E, Christos PJ, Souweidane MM (2008) Intraoperative assessment for endoscopic third 
ventriculostomy success. J Neurosurg Pediatr 2(5):298-303. https:// doi.org/10.3171/PED.2008.2.11.298

21. Romero L, Ros B, Ibáñez G, Ríus F, González L, Arráez M (2014) Endoscopic third ventriculostomy: can we predict success during surgery? Neurosurg Rev 37(1):89-97. https://doi.org/10.1007/ s10143-013-0494-6

22. Cinalli G, Spennato P, Nastro A, Aliberti F, Trischitta V, Ruggiero C, Mirone G, Cianciulli E (2011) Hydrocephalus in aqueductal stenosis. Childs Nerv Syst 27(10):1621-1642. https://doi.org/10. 1007/s00381-011-1546-2

23. Schmitt P, Jane JJ (2012) A lesson in history: the evolution of endoscopic third ventriculostomy. Neurosurg Focus 33(2):E11. https://doi.org/10.3171/2012.6.FOCUS12136

24. Teo C, Young R 2nd (1999) Endoscopic management of hydrocephalus secondary to tumors of the posterior third ventricle. Neurosurg Focus 15;7(4):e2

25. Shono T, Natori Y, Morioka T, Torisu R, Mizoguchi M, Nagata S, Suzuki SO, Iwaki T, Inamura T, Fukui M, Oka K, Sasaki T (2007) Results of a long-term follow-up after neuroendoscopic biopsy procedure and third ventriculostomy in patients with intracranial germinomas. J Neurosurg 107(3 Suppl):193-198. https://doi.org/ 10.3171/PED-07/09/193

26. Tamburrini G, Pettorini BL, Massimi L, Caldarelli M, Di Rocco C (2008) Endoscopic third ventriculostomy: the best option in the treatment of persistent hydrocephalus after posterior cranial fossa tumour removal? Childs Nerv Syst 24(12):1405-1412. https://doi. org/10.1007/s00381-008-0699-0

27. Roujeau T, Di Rocco F, Dufour C, Bourdeaut F, Puget S, Rose CS, Zerah M (2011) Shall we treat hydrocephalus associated to brain stem glioma in children? Childs Nerv Syst 27(10):1735-1739. https://doi.org/10.1007/s00381-011-1538-2

28. Stovell MG, Zakaria R, Ellenbogen JR, Gallagher MJ, Jenkinson MD, Hayhurst C, Mallucci CL (2016) Long-term follow-up of endoscopic third ventriculostomy performed in the pediatric population. J Neurosurg Pediatr 17(6):734-738. https://doi.org/10.3171/ 2015.11.PEDS15212

29. Drake J, Chumas P, Kestle J, Pierre-Kahn A, Vinchon M, Brown J, Pollack IF, Arai H (2006) Late rapid deterioration after endoscopic third ventriculostomy: additional cases and review of the literature.
J Neurosurg 105(2 Suppl):118-126. https://doi.org/10.3171/ped. 2006.105.2.118

30. Lam S, Harris D, Rocque BG, Ham SA (2014) Pediatric endoscopic third ventriculostomy: a population-based study. J Neurosurg Pediatr 14(5):455-464. https://doi.org/10.3171/ 2014.8.PEDS13680

31. Mobbs RJ, Vonau M, Davies MA (2003) Death after late failure of endoscopic third ventriculostomy: a potential solution. Neurosurgery 53(2):384-385; discussion 385-386. https://doi.org/ 10.1227/01.NEU.0000073534.04767.B1

32. Hader WJ, Drake J, Cochrane D, Sparrow O, Johnson ES, Kestle J (2002) Death after late failure of third ventriculostomy in children. Report of three cases. J Neurosurg 97(1):211-215. https://doi.org/ 10.3171/jns.2002.97.1.0211

33. Emery JL, Staschak MC (1972) The size and form of the cerebral aqueduct in children. Brain 95(3):591-598. https://doi.org/10.1093/ brain/95.3.591

34. Kulkarni AV, Sgouros S, Constantini S, Investigators IIHS (2016) International infant hydrocephalus study: initial results of a prospective, multicenter comparison of endoscopic third ventriculostomy (ETV) and shunt for infant hydrocephalus. Childs Nerv Syst 32(6):1039-1048. https://doi.org/10.1007/ s00381-016-3095-1

35. Tewuerbati S, Maimaitili M, Zhu G, Du G, Liu B, Sailike D, Fan Y, Dangmurenjiafu G (2015) Timing of endoscopic third ventriculostomy in pediatric patients with congenital obstructive hydrocephalus: assessment of neurodevelopmental outcome and short-term operative success rate. J Clin Neurosci 22(8):12921297. https://doi.org/10.1016/j.jocn.2014.12.030

36. Weil AG, Westwick H, Wang S, Alotaibi NM, Elkaim L, Ibrahim GM, Wang AC, Ariani RT, Crevier L, Myers B, Fallah A (2016) Efficacy and safety of endoscopic third ventriculostomy and choroid plexus cauterization for infantile hydrocephalus: a systematic review and meta-analysis. Childs Nerv Syst 32(11):2119-2131. https://doi.org/10.1007/s00381-016-3236-6

37. Baird LC (2016) First treatment in infants with hydrocephalus: the case for endoscopic third Ventriculostomy/choroid plexus cauterization. Neurosurgery 63(Suppl 1):78-82. https://doi.org/10.1227/ NEU.0000000000001299 